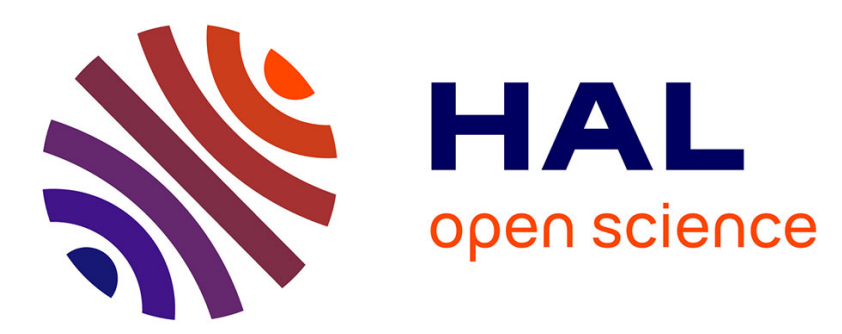

\title{
Évaluer la démocratisation de l'enseignement : la situation française à l'épreuve des comparaisons internationales
}

Marie Duru-Bellat, Annick Kieffer

\section{- To cite this version:}

Marie Duru-Bellat, Annick Kieffer. Évaluer la démocratisation de l'enseignement: la situation française à l'épreuve des comparaisons internationales. Revue Française de Pédagogie, 1999, 127, pp.49-61. hal-03209418

\section{HAL Id: hal-03209418 \\ https://hal.science/hal-03209418}

Submitted on 25 Jun 2021

HAL is a multi-disciplinary open access archive for the deposit and dissemination of scientific research documents, whether they are published or not. The documents may come from teaching and research institutions in France or abroad, or from public or private research centers.
L'archive ouverte pluridisciplinaire HAL, est destinée au dépôt et à la diffusion de documents scientifiques de niveau recherche, publiés ou non, émanant des établissements d'enseignement et de recherche français ou étrangers, des laboratoires publics ou privés.

\section{(ㅇ)(1) $\$$}

Distributed under a Creative Commons Attribution - NonCommercial - NoDerivatives| 4.0 


\title{
Evaluer la démocratisation de l'enseignement : la situation française à l'épreuve des comparaisons internationales
}

\author{
Marie Duru-Bellat \\ Annick Kieffer
}

La démocratisation de l'enseignement est une question délicate, d'un point de vue méthodologique aussi bien que conceptuel, et ce d'autant plus que des comparaisons internationales sont visées. Faut-il en effet analyser ce processus comme la diffusion d'un bien, ou s'attacher davantage à la compétition entre groupes sociaux qui en régit l'accès? Les indicateurs retenus ne seront pas les mêmes, ni bien sûr les résultats. Des comparaisons européennes montrent que si la majorité des pays ont choisi de démocratiser l'accès à l'éducation en ouvrant les portes de l'école, moins nombreux sont ceux qui ont tenté d'agir plus directement sur les inégalités qui marquent son fonctionnement. La France a joué avec retard cette carte de l'ouverture, notamment parce qu'ont subsisté longtemps des filières distinctes au sein du collège. Mais elle l'a jouée avec vigueur, notamment ces dernières années, tout en se situant à un niveau d'inégalité plus fort qu'en Grande-Bretagne ou qu'en Suède. Néanmoins, la France serait moins inégalitaire que ce dernier pays, quant à l'accès au Supérieur, précisément du fait de la forte expansion de ce niveau. Mais l'ouverture, voie a priori simple pour démocratiser, n'en pose pas moins un certain nombre de problèmes.

\footnotetext{
'analyse comparative des inégalités sociales d'accès et de réussite en éducation constitue un champ bien développé, non dépourvu de portée politique, comme en atteste l'abondance des publications réalisées sur ce thème par l'OCDE notamment. Mais la recherche française y apparaît peu présente. Par exemple, le recueil de travaux publié par Shavit et Blossfeld en 1993 sous le titre "Persistent Inequality" concerne treize pays, du Japon à la Pologne, mais pas la France. D'autres travaux comparatifs intègrent la France (par exemple ceux de Müller et Karle, 1993), mais
}

ils ne sont jamais conduits par des chercheurs français et leurs conclusions apparaissent parfois discutables, nous y reviendrons.

Cette discrétion française peut apparaître paradoxale, alors même que l'analyse des inégalités face à l'éducation constitue depuis les années 60 (avec les travaux fondateurs de Girard et Bastide, puis les constructions théoriques de Bourdieu et Passeron) un domaine privilégié par les sociologues. Tout se passe comme si le paradigme dominant avait conduit les chercheurs, dans leur 
majorité, à considérer a priori l'école comme l'instrument unique de la reproduction sociale dans une société divisée, et à négliger l'analyse empirique des relations entre titres scolaires et positions sociales, laissée aux spécialistes de la mobilité sociale. Cette coupure originelle entre sociologie de l'éducation et sociologie de la mobilité sociale, dans la mesure où c'est dans ce second type de réseau que se sont développées les comparaisons internationales, constitue sans doute une des raisons majeures de la faible présence française.

La recherche dont cet article rend compte a eu explicitement pour objectif d'élaborer sur la France des analyses les plus comparables possibles avec les travaux européens existants en matière de démocratisation des chances scolaires. Pour ce faire, nous nous sommes appuyées sur les 4 dernières enquêtes "Formation-Qualification" (FQP) de I'INSEE, réalisées en France en 1970, 1977,1985 et 1993 , ainsi que sur les trois panels successifs du Ministère de l'Éducation (suivant les scolarités d'élèves entrés en $6^{\circ}$ en $1972-73,1980$, et 1989), ce qui permet de prendre en compte des générations nées de 1919 à 1973 (sur les données FQP), et jusqu'aux années 1978, grâce au dernier panel (1).

Dans un premier temps, ceci a amené à "revisiter " la question largement explorée de la démocratisation de l'enseignement en France, avant de pouvoir construire des comparaisons. Dans ce texte, nous évoquerons assez rapidement les résultats produits sur la France, ainsi que les problèmes de méthode afférents, avant de présenter de manière plus précise les conclusions de certaines comparaisons.

\section{CONSTRUIRE DES COMPARAISONS : QUELQUES DIFFICULTÉS CONCEPTUELLES ET MÉTHODOLOGIQUES, À TRAVERS LE PRISME FRANÇAIS}

Pour engager cette analyse de la démocratisation du système éducatif, partons de l'évolution globale des scolarisations sur la période étudiée. Rappelons que celle-ci a été marquée par des bouleversements notables, dont le graphique ciaprès présente les plus importants. Si l'accès à la

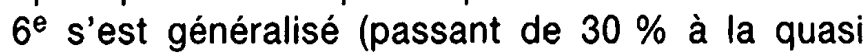

totalité d'une classe d'âge), l'évolution a été décalée (en calendrier et en intensité) concernant la classe de $3^{e}$, ceci étant encore plus vrai aux niveaux ultérieurs. Si l'accès à une $3^{\mathrm{e}}$ s'est développé du fait même de la généralisation de l'entrée en $6^{e}$, cette évolution a été contrariée par le développement concomitant de la sélectivité au sein même du collège : au fur et à mesure qu'y entraient plus d'élèves, l'orientation en fin de $5^{\mathrm{e}}$ vers des filières pré-professionnelles s'est développée, et au total, comme le montre le graphique, les taux d'accès en $3^{e}$ parmi les élèves entrés en $6^{\ominus}$ ont accusé une certaine baisse, notamment dans les années où l'accès à la $6^{6}$ se développait le plus, pour ne remonter que dans la période récente. De même, sur la période, les taux d'entrée en $2^{\text {de }}$ ont certes augmenté, de par le développement des scolarités dans le secondaire, mais de manière modérée du fait d'une sélectivité accrue lors du passage $3^{e} / 2^{\text {de }}$ (ce mouvement ne se renversant, là encore, que dans les générations les plus récentes).

Dans ce contexte, qu'en est-il de la démocratisation ? Elle est nette pour l'entrée en $6^{\mathrm{e}}$, en raison d'" effets de plafond " mécaniques : les taux d'accès des enfants de cadres étaient déjà proches de $100 \%$ en début de période, ceux des enfants de milieu populaire n'ont pu que s'en rapprocher. Par contre, au niveau de l'entrée en $2^{\text {de, }}$ la démocratisation apparaît très limitée jusqu'aux générations les plus jeunes (nées en 1964-73), car le développement des orientations en cours de collège, parallèlement à l'ouverture de la $6^{\mathrm{e}}$, a concerné avant tout les enfants de milieu populaire, ces nouveaux entrants y faisant donc des carrières plus souvent écourtées (les travaux sur l'orientation ont largement attesté du caractère très biaisé socialement du palier d'orientation de la fin de la $5^{e}$; cf. Duru-Bellat et al., 1993). Ce n'est que très récemment, avec la suppression de ce palier d'orientation et le développement corollaire des scolarités complètes au collège, que les inégalités sociales d'accès à une classe de $2^{\text {de }}$ amorcent une baisse significative, que fait apparaître la comparaison des panels 80 et 89 . Mais il faut souligner que les inégalités inhérentes au passage $3^{\mathrm{e}} / 2^{\text {de }}$ semblent relativement stables. Ainsi, pour les enfants d'ouvriers, la comparaison des panels 80 et 89 montre que si le gain dans l'accès à une $3^{e}$ est de 31 points (les taux passent de 58 à $89 \%$ ), le gain est moins que proportionnel quand on se centre sur l'accès à une $2^{\text {de }}$ 


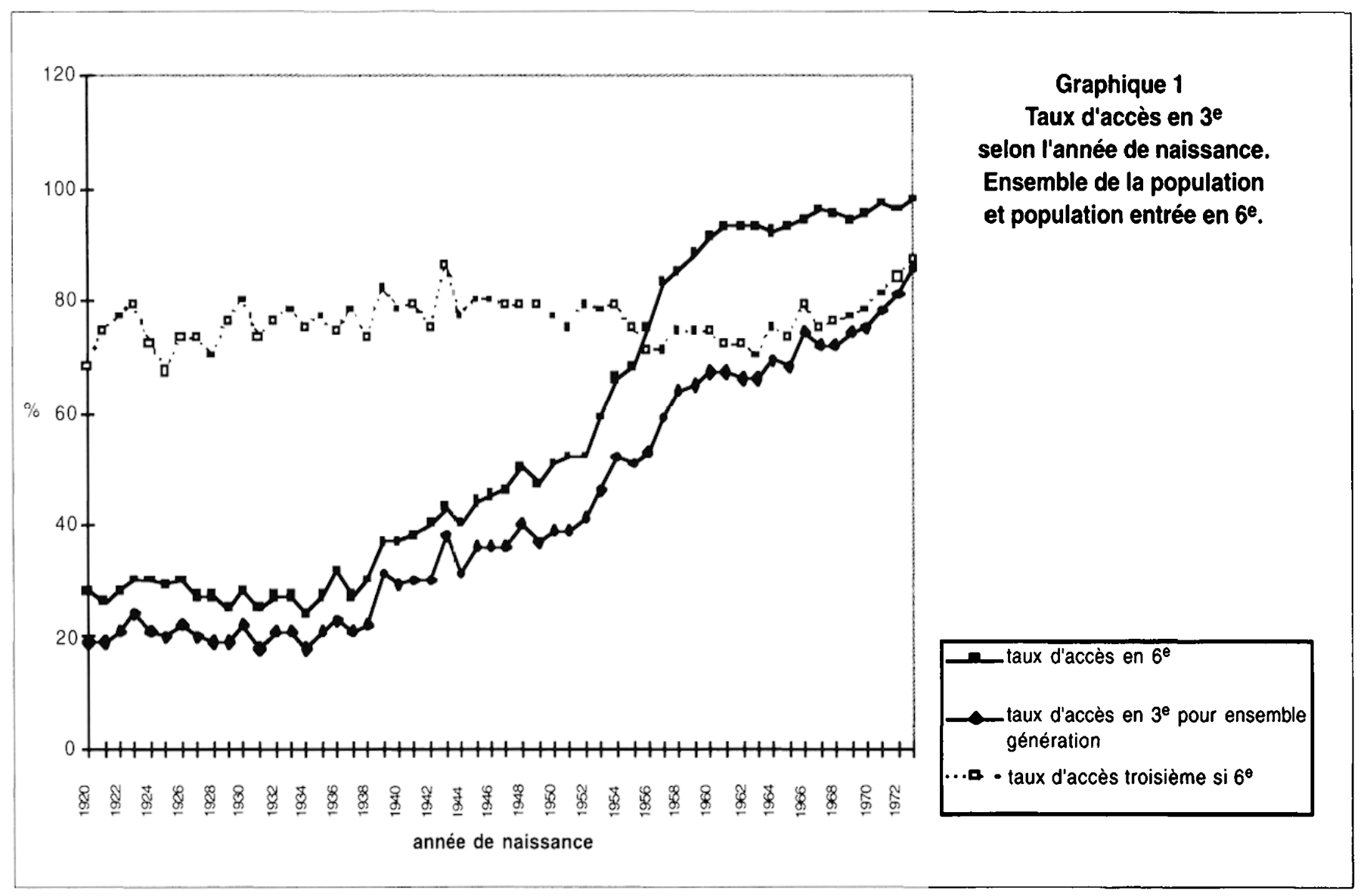

générale ou technologique (les taux étant respectivement de 30 et $42 \%$ ). Tout comme la démocratisation de l'accès à une $3^{\mathrm{e}}$ était entièrement "tirée " par la généralisation de l'entrée en $6^{e}$ (sans que l'orientation en cours de collège se soit démocratisée), la démocratisation récente de l'entrée au lycée résulte essentiellement de l'ouverture des niveaux antérieurs.

L'appréhension de ces grandes tendances peut apparaître relativement immédiate. Pourtant, la comparaison de nos résultats avec les diverses recherches réalisées sur cette question illustre un certain nombre de problèmes indissociablement méthodologiques et conceptuels. Tout d'abord, il convient de bien spécifier les taux d'accès mobilisés pour suivre l'évolution. Ainsi, quand Prost (1986) indique que dans la période 1967-1980, la démocratisation semble étale en ce qui concerne l'accès au second cycle, il faut bien voir que la stabilité des taux d'accès au lycée dans les milieux populaires cache un double mouvement: une légère hausse des taux d'accès (résultant du développement des scolarités en amont), mais contrariée par l'accroissement de la sélectivité des carrières. La distinction que nous avons faite systématiquement entre taux d'accès globaux et taux d'accès "si $6^{\theta}$ " n'a donc pas un caractère accessoire. Elle renvoie en fait à deux facettes de la démocratisation, par ailleurs bien perçues intuitivement par Prost, ce qu'il appelle la " démographisation " (démocratisation quantitative, découlant mécaniquement de l'ouverture de l'accès à un niveau) et une démocratisation plus qualitative, qui traduirait un déroulement des carrières moins dépendant de l'origine sociale (soit que les réussites soient moins inégales, soit que s'estompent des inégalités d'orientation à réussite comparable).

De même, le mode de confrontation des taux n'est pas sans importance. Si de nombreux débats ont pris place en France sur la manière d'appréhender les évolutions (cf. notamment Combessie, 1984 ; Merllié, 1985), les chercheurs en éducation continuent à utiliser sans justification particulière soit des différences de pourcentages, soit des rapports entre pourcentages ou des taux de croissance. Ainsi, Langouët (1994), dans sa comparaison des panels 72 et 80 , conclut-il à une démocratisation de l'accès au niveau $4^{e}$, sur la seule base de l'examen des taux 
de variation des taux respectifs des enfants de cadres et d'ouvriers ; les débats évoqués ci-avant convainquent pourtant que ceci n'est pas sans rapport avec la méthode retenue, les taux de variation amenant systématiquement à conclure à une réduction des inégalités dans les périodes de croissance des taux (2). Nos calculs, fondés sur le calcul de "odds ratios" (3) débouchent sur le constat d'une absence totale de réduction des inégalités à ce niveau et sur cette période.

En fait, ces divergences ne sont pas purement techniques, car le choix d'un indicateur s'articule avec la définition de l'égalité des chances et de la démocratisation que le chercheur privilégie. Si l'on considère avant tout l'éducation comme un bien en soi (indépendamment de sa possession par d'autres), on se centrera sur l'accès à l'école, et toute ouverture du système sera considérée en elle-même comme démocratisante. Le développement de la scolarisation (et donc la variation des " marges ", c'est-à-dire des taux moyens d'accès observés dans l'ensemble de la population à telle ou telle période) fait alors partie de l'analyse: c'est sur la base de différences entre taux d'accès, d'une catégorie sociale à l'autre, qu'on se demandera comment ont évolué les chances d'admission des uns et des autres. Même si les différences absolues entre taux sont très sensibles aux distributions des taux moyens (en particulier, elles se réduisent d'autant plus que les taux se rapprochent de $100 \%$ ), elles seront centrales dans l'analyse, dans la mesure où elles informent sur les ressemblances/dissemblances des expériences éducatives des jeunes des différents milieux sociaux. Cette perspective semble avoir de fait dominé les travaux sur la démocratisation conduits en France (les rendant peu propices aux comparaisons internationales).

Mais on peut choisir de mettre en avant la fonction instrumentale de l'éducation (toute formation n'ayant de valeur que distinctive, l'objectif étant de se classer pour accéder à des positions sociales hiérarchisées), perspective privilégiée par les sociologues étrangers centrés le plus souvent sur la problématique de la mobilité sociale; c'est alors la compétition entre groupes qui est essentielle. Pour évaluer l'écart des chances scolaires entre telle ou telle catégorie sociale, la prise en compte des marges (l'évolution des taux d'accès à tel ou tel niveau dans l'ensemble de la population) est alors inutile, voire trompeuse, et on privilégiera des indicateurs "insensibles aux marges ", moins dépendants du niveau absolu des taux, de type " odds ratio " (différences relatives). Notons que dans cette seconde perspective est mise en exergue l'importance de l'offre en matière de formation dans l'ampleur des inégalités sociales. C'est en effet l'offre de places qui fixe le seuil entre les "sélectionnés" et les autres, et donc la dureté de la sélection (notamment sociale) qui va alors prendre place.

Les comparaisons dans le temps et dans l'espace sont donc bien à construire en fonction d'indicateurs qui traduisent une certaine conception de la démocratisation. Pour défendre véritablement le choix de telle mesure, il faudrait " pouvoir formuler et justifier un jugement de pertinence sur des hypothèses associées explicitement à chacune de ces mesures" (Combessie, 1984). Toujours est-il qu'une exigence minimale pour le chercheur est d'expliciter ses choix et de s'efforcer d'utiliser l'indicateur qui apparaît dans tel contexte comme le plus pertinent.

\section{ESQUISSE DE QUELQUES COMPARAISONS EUROPÉENNES}

Pour construire ces comparaisons dans le temps et dans l'espace, il est apparu préférable de privilégier des indicateurs relativement insensibles aux marges de type odds ratios (ou encore, ce qui revient au même, des modèles de type logistique). En effet, tous les chiffres concernant les scolarités des jeunes et les inégalités qui les marquent sont à l'évidence datés et situés. Datés, car ils font suite (en France, mais aussi dans la totalité des pays pris en compte ici) à une période d'expansion des scolarités, qui a concerné plus ou moins les différents segments du système éducatif. On peut s'attendre à ce que cette expansion débouche mécaniquement sur une réduction des inégalités sociales, dès lors que le taux d'accès à un niveau se rapproche de $100 \%$, des effets de plafond s'observant alors dans les groupes qui y accédaient déjà le plus souvent. Le niveau brut d'inégalités constaté dans un pays, concernant l'accès à tel ou tel niveau éducatif, est donc lié à la fréquence d'accès de l'ensemble de la population d'élèves concernés à ce niveau. On ne peut donc comparer sans précautions deux situations ou deux sites caractérisées par une distribution différente dans l'accès à tel ou tel "bien " ou niveau 
éducatif. II convient aussi d'être également très vigilant sur le caractère situé des chiffres. Ceux-ci ne prennent leur sens qu'au sein d'un pays donné, par rapport à la structure formelle du système éducatif à tel ou tel moment, et aux hiérarchies plus ou moins informelles entre filières (4).

Nous nous centrerons ici : 1) sur le plus haut niveau atteint, 2) sur quelques transitions stratégiques dans tous les systèmes : l'entrée en $6^{e}$, l'entrée en $2^{\text {de }}$, et l'entrée dans l'enseignement supérieur, en mobilisant essentiellement des données concernant l'Allemagne, l'Italie, la Suède, et la Grande-Bretagne.

\section{Une première approche synthétique des inégalités}

Une première approche des inégalités et de leur évolution au cours du siècle consiste à estimer des modèles de régression mettant en regard le plus haut niveau éducatif atteint (traité comme une variable continue) et des variables caractérisant l'origine sociale de l'individu, ceci par groupes de générations. Dans ce cas, le pourcentage de la variance du niveau éducatif $\left(R^{2}\right)$ expliquée par ces variables constitue un indicateur simple de la force des inégalités sociales face à l'éducation; il renseigne, en d'autres termes, sur la valeur du pronostic, quant au niveau d'éducation qu'atteindra un individu, que l'on peut faire sur la base de l'information fournie sur son origine sociale. Cet indicateur est globalement comparable dans le temps et dans l'espace, bien que la valeur du $R^{2}$ ne soit pas insensible à la manière dont les données sont construites (période de temps prise en compte, finesse des nomenclatures, etc.). II apparaît néanmoins que les inégalités face à l'éducation sont plus restreintes en Grande-Bretagne (sur l'ensemble de la période) et aux Pays-Bas (en fin de période) qu'en France. L'Italie et l'Allemagne présentent un niveau global d'inégalité légèrement supérieur à la France.

Ce tableau fait également apparaître une baisse de la force de l'association entre origine sociale et niveau éducatif atteint. On observe aussi que dans la majorité des pays pour lesquels les données existent, c'est l'influence du niveau d'instruction du père (ou des parents, le niveau d'instruction de la mère étant assez souvent pris en compte) qui a baissé, l'influence de la profession paternelle (à niveau d'instruction contrôlé) étant quant à elle beaucoup plus stable.

Ces évolutions restent néanmoins d'interprétation délicate. On sait en effet que les régressions du plus haut niveau éducatif atteint peuvent être trompeuses dans un contexte historique de développement de la scolarisation (Mare, 1981). L'évolution des inégalités que révèlent les régressions tient à la fois : 1) au développement des taux d'accès aux différents niveaux, du fait de

\section{Tableau 1. - Évolution des relations entre niveau éducatif atteint et origine sociale dans quelques pays européens}

\begin{tabular}{|l|c|c|c|l|}
\hline Pays & $\begin{array}{c}\text { Cohortes } \\
\text { concernées }\end{array}$ & $\begin{array}{c}\text { Niveau d'instruction } \\
\text { du père }\end{array}$ & $\begin{array}{c}\text { Profession } \\
\text { du père }\end{array}$ & Évolution du R2 $\left(^{*}\right)$ \\
\hline Allemagne & $1916-1965$ & 0 & 0 & $25 \%$ à $28 \%$ effet moyen sur la période \\
\hline Grande-Bretagne & $1913-1952$ & 0 & 0 & $17,7 \%$ effet moyen sur la période \\
\hline France & $1920-1973$ & - & 0 & $27,6 \%$ effet moyen sur la période \\
\hline Italie & $1920-1961$ & - & 0 & de $46-39 \%$ (déb.) à $28-26 \%$ (fin) \\
\hline France & $1920-1973$ & - & 0 & de $32,3 \%$ (déb.) à $20,3 \%$ (fin) \\
\hline Pays-Bas & $1891-1960$ & - & - & de $31-45 \%$ (déb.) à $12 \%$ (fin) \\
\hline
\end{tabular}

+ = association croissante, 0 = stabilité, - = association décroissante.

(") Cette information (pourcentage de la variance du niveau éducatif atteint expliqué par l'origine sociale et la cohorte) n'est pas disponible dans tous les pays. En cas de fourchette, il s'agit de chiffres fournis séparément pour les hommes, puis pour les femmes. 
l'expansion du système, qui entraîne tendanciellement une baisse des inégalités dès lors que la scolarisation tend à devenir universelle ; 2) à l'association entre origine sociale et accès à l'éducation, deux phénomènes qui peuvent être en partie indépendants: l'expansion du système peut en elle-même entraîner une certaine atténuation des inégalités sans que l'association entre carrière scolaire et milieu social ait été modifiée.

Si observer que, du fait de son développement, l'éducation est un bien moins inégalitairement partagé n'est pas dénié de pertinence sociologique, il est clair qu'une approche comparative des inégalités que produit le fonctionnement des systèmes éducatifs étudiés doit se centrer sur la force de l'association entre origine sociale et carrière scolaire. On est alors conduit à travailler sur les transitions qui jalonnent la carrière scolaire des élèves, en construisant des modèles logistiques expliquant les probabilités d'accès aux niveaux successifs, en fonction de l'origine sociale et de la génération d'appartenance.

\section{L'entrée dans le premier cycle de l'enseignement secondaire}

Le passage primaire/secondaire a l'avantage d'être relativement comparable d'un pays à l'autre; ce palier d'orientation est par contre amené, dans la plupart des pays, à devenir obsolète, dès lors qu'ont été mises en place, le plus souvent à partir de l'après guerre, des réformes éducatives rendant obligatoire l'accès au secondaire, au sein d'une structure en général unifiée. Certains pays ont néanmoins gardé un système à filières (tels que l'Allemagne, l'Autriche, la Suisse ou le Luxembourg), ce qui rend moins immédiate l'appréhension des inégalités à ce niveau. Ainsi, en Allemagne, une proportion importante d'enfants aborde une formation professionnelle à la fin de la Hauptschule, qui correspond à nos anciennes classes de fin d'études primaires; dans ce cas, seule l'entrée dans les filières menant aux niveaux moyens et supérieurs du secondaire (l'entrée en Realschule ou en Gymnasium) pourrait être comparable à l'accès à une classe de $6^{e}$ en France ou dans les pays à système " compréhensif ". Les allemands utilisent peu cet indicateur, mais on dispose de données sur la filière fréquentée par les élèves de 13 ans, en 1976 et 1989 en fonction de l'origine sociale (Kölher, 1992).

Bien que la nomenclature des professions ne permette pas des comparaisons directes avec des données françaises (les Beamten recouvrant l'ensemble des fonctionnaires, catégorie plus restreinte et plus diplômée qu'en France, alors que les Angestellten recouvrent une population plus large que les cadres, incluant jusqu'aux employés), on peut utiliser cet indicateur d'inégalité qu'est le odds ratio (rappelons qu'il s'agit d'un indice de chance relative, indépendant du niveau absolu des chiffres). Les chiffres montrent que l'accès au Gymnasium est marqué par de

Tableau 2. - Ventilation des enfants de cols blancs et des enfants d'ouvriers âgés de 13-14 ans en 1976 et 1989 selon les principales filières (en \%).

\begin{tabular}{|l|c|c|c|c|c|c|}
\hline & \multicolumn{2}{|c|}{ Hauptschule } & \multicolumn{2}{c|}{ Realschule } & \multicolumn{2}{c|}{ Gymnasium } \\
\cline { 2 - 7 } & 1976 & 1989 & 1976 & 1989 & 1976 & 1989 \\
\hline Beamte (fonctionnaires) & 25,7 & 14,0 & 22,5 & 24,2 & 47,4 & 57,4 \\
\hline Angestellte (cols blancs) & 32,1 & 22,1 & 24,7 & 29,8 & 37,8 & 42,8 \\
\hline Arbeiter (ouvriers) & 64,2 & 58,3 & 19,5 & 26,0 & 9,3 & 10,8 \\
\hline Ensemble de la population & 50,1 & 39,6 & 21,4 & 26,8 & 22,5 & 28,7 \\
\hline $\begin{array}{l}\text { odds-ratios (a) Beamte } \\
\text { vs ouvriers }\end{array}$ & 0,19 & 0,12 & 1,20 & 0,91 & 8,8 & 11,1 \\
\hline $\begin{array}{l}\text { odds-ratios (b) Angestellte } \\
\text { vs ouvriers }\end{array}$ & 0,26 & 0,20 & 1,35 & 1,21 & 5,9 & 6,2 \\
\hline
\end{tabular}

Calculs effectués à partir de Köhler (1992). 
sensibles inégalités, qui ont eu plutôt tendance à se renforcer dans la période récente; à l'inverse, la Hauptschule est particulièrement délaissée par les catégories favorisées et semble devenir de plus en plus un ghetto pour enfants d'ouvriers. $\mathrm{Si}$ on additionne les chiffres concernant la Realschule et le Gymnasium de manière à obtenir quelque chose de comparable à l'entrée en $6^{e}$, on obtient des odds ratios élevés et qui ont plutôt tendance à s'accentuer (si on calcule, en fin de période, le rapport de chances entre ces deux groupes, sur la base du total $24,2+57,4$ versus $26,0+10,8$, on obtient pour l'indicateur a un odd ratio de 7,6$)$. En France, aux périodes considérées (correspondant globalement aux panels 80 et 89 ), cet indicateur est proche de 1 (qui indique une égalité des chances relatives) pour l'entrée en $6^{\mathrm{e}}$ (puisque les taux d'accès sont pratiquement égaux à 100\%); par contre, pour l'accès à une classe de $4^{e}$ générale (comparaison justifiée, car les deux filières allemandes considérées ne prévoient pas de palier d'orientation au bout de deux ans), les odds ratios opposant enfants de cadres et enfants d'ouvriers sont de 19,6 pour le panel 80 et de 9,4 pour le panel 89 . Les chiffres français sont donc plus élevés, mais ils sont nettement en baisse, et deviennent en fin de période comparables aux odds ratios calculés pour l'accès au seul Gymnasium $(11,1)$. La France serait donc en passe de devenir moins inégalitaire à ce niveau, sans doute non sans rapport avec les changements structurels qui ont pris place, alors que le système allemand se caractérise par une grande stabilité institutionnelle.

En Grande-Bretagne, alors même que le niveau global d'inégalités face à l'école apparaît relativement modéré, l'accès à l'école secondaire est resté, jusqu'aux générations nées au début des années 50 , le niveau le plus sélectif socialement, par le biais du type d'école fréquentée. En l'occurrence, les élèves entrant dans une école sélective (Grammar school ou école privée) réalisent des carrières scolaires significativement plus longues que ceux qui accèdent à des écoles non sélectives (Secondary-modern ou Comprehensive schoo/s). Ce n'est que dans les années 70 que les Comprehensive schools sont devenues majoritaires en Grande-Bretagne, avec à la clé une certaine réduction des inégalités.

Dans des pays comme la Suède ou l'Italie, l'accès à l'" école moyenne " s'est généralisé assez tôt. En Suède, les taux d'accès des enfants de milieu aisé étaient déjà proches de $100 \%$ dans les générations nées au début du siècle (1902-1909), alors que seulement un cinquième des enfants de milieu défavorisé y accédaient alors. Une politique d'unification et d'ouverture du système a été menée tout au long des années 1930-50 (la Grundskola qui accueille les enfants de 7 à 16 ans ayant été instaurée en 1962); effets de plafond aidant, les inégalités se sont sensiblement réduites (avec des taux d'accès proches de $80 \%$ chez les enfants de milieu défavorisé parmi les générations nés en 1961). En Italie, l'égalisation aurait pris place avant même les réformes de 1963 portant sur la Scuola media (instaurant l'école unique), et aurait concerné les générations nées dans les années 40. En France, rappelons qu'il faut attendre l'application de la réforme Berthoin (à partir de 1967 , et ne touchant intégralement que les générations nées à partir des années 1955) pour que les taux d'accès des enfants d'ouvriers s'élèvent sensiblement, les odds ratios ne baissant fortement qu'à partir des générations 1959-63. La France se distingue donc par un calendrier un peu décalé eu égard à la généralisation de l'accès à l'école moyenne, qui a pris place dans tous les pays européens (à l'exception de ceux ayant gardé des systèmes à filières). On comprend alors que les comparaisons internationales, qui se sont le plus souvent fondées pour la France sur les générations nées avant les années 50 (5) n'aient pu prendre en compte ces évolutions et aient conclu à l'existence d'inégalités particulièrement importantes dans notre pays.

Mais plus significatif encore de la situation française est le maintien de filières diversifiées au collège (entre 1967 et 1978), avec même une accentuation de la sélectivité, nous l'avons évoqué, dans les années 70 . L'école moyenne à la française a ainsi pu fonctionner de manière assez comparable au système allemand à filières ou au système anglais à plusieurs types d'école.

\section{L'accès au " secondaire supérieur "}

Au terme de cette école moyenne plus ou moins unifiée, de nouvelles inégalités apparaissent lors du passage au lycée (soit, dans les nomenclatures internationales, du secondaire inférieur au secondaire supérieur, sachant que dans une partie de ces travaux, l'accès au secondaire supérieur n'est pas toujours distingué de l'obtention du diplôme de fin de cycle). Des comparaisons 
internationales (Jonnson, Mills et Müller, 1996), portant sur les générations 1950-1959, donnent à ce niveau de transition les odds ratios suivants : 6,2 pour la Suède, 7,5 pour la Grande-Bretagne, 9,6 pour l'Allemagne, et nous obtenons pour la France un chiffre de 9 (en comparant enfants de cadres et ouvriers, mode de calcul imparfaitement comparable à celui retenu dans les autres pays, qui opposent les enfants de la "service class" aux enfants de l'" unskilled working class", cf. note 3).

La France apparaît néanmoins comme dans une situation plus inégalitaire que la Suède ou la Grande-Bretagne, et assez proche de la situation allemande. On peut à ce propos noter que l'Allemagne fait preuve d'un niveau d'inégalité sociale comparable à la France par le jeu de mécanismes différents : orientation précoce entre trois voies étanches dès la sortie du primaire, avec ensuite un cursus entier dans la filière, alors que la configuration française apparaît plus égalitaire au niveau $6^{\mathrm{e}}$, mais laisse la porte ouverte à des carrières socialement différenciées au sein même d'une structure apparemment unique. De manière comparable, la Grande-Bretagne qui apparaissait relativement inégalitaire par la coexistence de types d'écoles distincts, se " rattrape " en quelque sorte par le fonctionnement de l'orientation vers le secondaire supérieur ; celui-ci est en l'occurrence plus " méritocratique " qu'en France, puisque fondé sur l'obtention des résultats obtenus au diplôme de fin du secondaire inférieur (GCSE O-level), alors que dans notre pays, le poids donné aux demandes familiales constitue une source autonome d'inégalités sociales à chacun des paliers d'orientation (DuruBellat et al., 1993).

Mais à nouveau, à ce stade de la scolarité, la France apparaît un peu "en retard " par rapport à des pays comme la Suède ou l'Italie; dans ce dernier pays par exemple, une égalisation significative de l'accès au lycée a concerné les générations nées entre 1930 et 1950 , en partie du fait d'effets de plafond, les taux d'accès des enfants des groupes les plus instruits étant déjà très élevés en début de période. Rappelons qu'en France, par contraste, les taux de passage $3^{e} / 2^{\text {de }}$ des enfants d'ouvriers ont eu tendance à baisser entre les générations 1954-1963 (non sans lien avec le développement, à cette période, des filières de l'enseignement technique court), n'amorçant une hausse qu'à partir des générations 1964-1973.

\section{La quête de la distinction, par l'accès à l'enseignement supérieur, ou à des options spécifiques}

Venons-en enfin au niveau de l'accès au Supérieur, troisième "transition" importante, d'autant plus qu'à ce niveau, c'est l'accès à l'" élite" qui se joue. Ce niveau reste en général sélectif, même s'il s'est fortement développé dans la plupart des pays, de manière inégale toutefois (plus fortement en France ou en Italie qu'en Suède ou en Grande-Bretagne, par exemple). On dispose en l'occurrence d'un travail comparatif intéressant (Müller, 1996), mais avec à nouveau utilisation de données anciennes sur la France.

Sur cette base, la France apparaît comme un pays où à la fois les niveaux d'enseignement égaux ou supérieurs au bac seraient moins développés que dans les pays voisins, et où les inégalités sociales seraient particulièrement marquées. En fait, en estimant sur nos données des chiffres plus comparables aux sources étrangères et couvrant une période plus récente, on observe une expansion très sensible de l'enseignement supérieur, à tel point qu'à partir des cohortes 19591968, les chiffres concernant les enfants de cadres plafonnent, alors que ceux concernant les enfants d'ouvriers augmentent, ce qui amorce une égalisation.

L'expansion sensible par rapport aux pays voisins de l'enseignement supérieur en France, semble en elle-même avoir déjà produit une égalisation plus forte que dans des pays ayant eu une politique plus restrictive à ce niveau. C'est le cas notamment de la Suède: au début des années 90 , les taux d'obtention d'un diplôme du Supérieur sont, pour toutes les catégories sociales, supérieurs en France à ce qu'ils sont en Suède, vu l'inégal développement de ce niveau d'enseignement dans les deux pays. Sur les générations nées dans les années 50 , les odds ratios sont très proches ( 4,9 pour la Suède, 5,2 pour la France, avec un mode de calcul imparfaitement comparable toutefois), alors qu'on obtient un chiffre de 9,6 pour l'Allemagne. En Grande-Bretagne, les odds ratios sont très différents selon le type de filière du Supérieur, avec des inégalités sociales beaucoup plus fortes pour l'accès aux Universités traditionnelles $(12,9)$ que pour l'accès aux instituts 
"Polytechnics" $(3,9)$ ou aux "Colleges" $(3,4)$. Faut-il le rappeler, l'hétérogénéité sociale est encore plus forte en France, depuis les grandes écoles jusqu'aux sections de technicien supérieur ; pour ne citer qu'un chiffre, et d'après Euriat et Thélot (1995), le odds ratio s'élèverait jusqu'à 38 pour ce qui concerne l'accès à l'Ecole Polytechnique...

Si donc, au niveau du Supérieur comme aux niveaux précédents, l'ouverture du système entraîne immanquablement une certaine démocratisation, il faut compter avec les inégalités "qualitatives " qui tendent à se recréer sans cesse, au fur et à mesure que s'estompent les inégalités quantitatives. On sait par exemple qu'en Grande-Bretagne un certain nombre de différenciations fines de cursus, non neutres socialement, continuent d'exister même au sein des écoles "compréhensives", de même que des inégalités fines de réussite s'observent toujours (concernant par exemple l'obtention de notes assez élevées pour envisager de poursuivre l'étude d'une matière au niveau secondaire-supérieur). Le niveau académique de validation même des études, notamment par le biais des mentions obtenues, se substituerait donc à la dichotomie accès/non accès comme critère de distinction sociale. En France, si les enfants d'ouvriers ont été beaucoup plus nombreux à accéder à un baccalauréat scientifique au début des années 90 , les enfants de cadres auraient maintenu, voire creusé, leur avantage quant à la mention attachée à ce baccalauréat (Mear et Merle, 1992). On connaît également l'importance des options comme critère de distinction sociale: si en Grande-Bretagne, les enfants de milieu populaire sont, à niveau scolaire comparable, plus nombreux à choisir d'étudier les "vocational subjects " et moins nombreux à étudier les langues, en France, on connaît le caractère très typé socialement de l'étude des langues anciennes et de l'allemand. Au total, dans un contexte où l'essentiel est le classement, ces stratégies de positionnement scolaire de l'enfant par le jeu des options, sont de fait rationnelles, au moins tant que les diplômes restent dotés d'une valeur économique et sociale.

\section{LES COMPARAISONS INTERNATIONALES, UNE VOIE HEURISTIQUE POUR LA RECHERCHE}

Pour comprendre les différences dans le niveau, la structure et l'évolution des inégalités sociales face à l'école, d'un pays à l'autre, il est sans doute heuristique de recenser systématiquement les facteurs qui les engendrent et de déterminer ceux qui sont probablement les plus différenciateurs entre les pays. Cette réflexion est également heuristique pour comprendre les ressemblances que l'on observe dans la structure des inégalités : même si le déroulement global des carrières diffère, les inégalités sociales observées aux différentes transitions sont très proches (en particulier, ce sont toujours les mêmes groupes sociaux qui apparaissent avantagés). En ce qui concerne les inégalités de réussite, la sociologie tend à les expliquer par les modalités de la confrontation entre pratiques éducatives et cultures familiales d'une part, culture scolaire de l'autre. S'il est vrai en particulier que les contenus scolaires et les normes d'évaluation sont marqués par un "middle-class bias ", on ne voit pas pourquoi il y aurait de grandes variations d'un pays à l'autre (entre pays occidentaux comparables), dans l'intensité et la structure de ce type d'inégalités.

Les inégalités de passage ou plus largement d'orientation (avec, dans la plupart des pays, comme en France, une auto-sélection scolaire plus ou moins forte selon le milieu social, notamment chez les élèves faibles) sont a priori davantage susceptibles de variations entre pays, alors même que leur poids est fort dans les inégalités sociales de carrière scolaire. En effet, alors que les inégalités de réussite sont la conséquence de la socialisation passée et de l'exercice du "métier d'élève ", les inégalités d'orientation concernent l'anticipation de l'avenir. Or cette anticipation de l'avenir est elle-même fonction de multiples facteurs, susceptibles de varier selon les pays. On pense en particulier aux ressources de la famille, à la structure institutionnelle du système éducatif, aux incitations à la poursuite d'études.

Selon les pays, les inégalités "objectives" entre les classes, en termes de ressources et de sécurité économique, peuvent être plus ou moins marquées ; or ceci n'est pas sans influer sur la façon dont le jeune prend des décisions lors de transitions d'un niveau à l'autre et évalue leur niveau de risque, compte tenu de la perception qu'il a du soutien économique que sa famille est susceptible de lui apporter. Ainsi, en Suède, la baisse significative des inégalités sociales de carrières scolaires s'expliquerait notamment par l'élévation de la sécurité économique dans les milieux populaires, avec du 
même coup, des choix plus "tolérants au risque " de la part des jeunes de ces milieux sociaux.

Mais tout aussi déterminantes sont certaines caractéristiques structurelles du système éducatif au sein duquel ces choix sont à faire :

- L'ampleur de la sélectivité à chacune des transitions ; il s'agit là d'une des caractéristiques les plus cruciales des systèmes: l'ampleur des inégalités, dans un pays donné, dépend d'une part du niveau absolu de la scolarisation à chacun des niveaux (y a-t-il $20 \%$ d'une classe d'âge qui entre dans le secondaire supérieur, ou $80 \%$ ?), d'autre part du " survival pattern ", c'est-à-dire de la structure d'ensemble des taux de passages d'un niveau à l'autre. D'après les analyses de Müller et Karle (1993), les différences d'ampleur des inégalités sociales entre pays résulteraient avant tout des modalités dans l'organisation du système et de sa gestion des flux, et non de spécificités nationales dans la force de l'association entre origine sociale et origine scolaire.

Tous les pays l'ont d'ailleurs bien compris: une façon de démocratiser l'accès à l'éducation, c'est d'ouvrir le système, de développer l'offre (et donc de laisser passer davantage d'élèves aux différentes transitions), la démocratisation quantitative ayant un caractère mécanique, comme nous l'avons évoqué, dès lors que des "effets de plafond " se manifestent chez les classes aisées. C'est ainsi qu'en Suède (Jonsson, 1987 ; Jonsson et Mills, 1993), un objectif d'égalisation des chances scolaires, et de démocratisation de la société en général a amené à allonger la scolarité obligatoire et à réduire les points de sélection (les 9 premières années de scolarité étant communes à tous les élèves). C'est pour la même raison que, dans les comparaisons internationales fondées sur des données anciennes, la France apparaissait comme un des pays les plus inégalitaires : ceci tenait avant tout à la sélectivité globale très forte, tenant au "survival pattern " de l'époque, en l'occurrence à la structure duale du système (classes de fin d'études d'un côté, lycées de l'autre, où accédait une proportion limitée d'élèves), et non à la force particulière de l'association entre origine sociale et accès ou réussite aux différents niveaux. Par contraste, en Allemagne, le "survival pattern" dominé par l'importance du système de formation professionnelle (assimilé à l'enseignement secondaire du fait qu'il débouche sur une certification reconnue) serait moins sélectif socialement, mais ceci est contrebalancé par l'existence d'inégalités sociales (dans l'accès au Gymnasium notamment) plus marquées qu'en France (Müller et Karle, 1993). Ces exemples illustrent combien les inégalités devant l'école observées dans un pays donné, relèvent tout autant de facteurs institutionnels que de mécanismes individuels (Müller et Shavit, 1998).

- L'existence d'obstacles ou de choix précoces ; on sait (cf. les observations faites en Italie ou en Suède par exemple) que le fait de supprimer un obstacle précoce et de différer les choix atténue en général les inégalités; ceci se comprend dans la mesure où les inégalités sociales sont en général plus marquées aux premiers paliers d'orientation.

- Les règles gouvernant les passages d'un niveau à l'autre, avec davantage d'inégalités là où les choix des familles sont souverains (cf. DuruBellat et Mingat, 1992), et sans doute aussi là où le système apparaît fort complexe, déchiffrable par les seuls initiés.

- L'existence de filières d'élites, ou de types d'écoles distinctes, où vont se concentrer les enfants de milieux aisés, ou à l'inverse, l'existence de filières de relégation constituant des voies de garage (interdisant toute seconde chance), pour les enfants de milieu populaire.

Les réformes éducatives ont en général tenté d'agir sur ces différents paramètres, tout en privilégiant de fait l'ouverture du système, dont les effets démocratisants apparaissent garantis. Certes, une alternative, toujours pour égaliser, serait d'agir plus directement sur le fonctionnement du système, accompagnant ainsi la démocratisation quantitative d'une démocratisation qualitative, mais c'est à l'évidence plus complexe. Les comparaisons internationales montrent pourtant que rendre la sélection plus " objective ", plus méritocratique (via des tests de connaissance), par exemple, peut déboucher, comme c'est le cas au Royaume-Uni, sur un niveau d'inégalité sociale relativement faible; les performances des élèves prennent ainsi un poids relatif plus fort par rapport aux choix des familles, ce qui atténue l'auto-sélection sensible qui caractérise à cet égard les milieux populaires.

Enfin, qui dit choix dit anticipation de l'avenir; or, selon les pays, les incitations à poursuivre des études, autrement dit les bénéfices attendus de la scolarité, peuvent varier sensiblement. Car la manière dont s'articulent formations et emplois varie selon les contextes nationaux. Par exemple, l'effet du niveau d'instruction sur le prestige de la 
profession occupée est presque deux fois plus fort en Allemagne qu'en Grande-Bretagne, la France étant dans une situation intermédiaire (Müller et Shavit, 1998). Parmi les facteurs susceptibles d'expliquer ces variations, il y a (à nouveau) certains traits organisationnels inhérents au système scolaire. Les relations entre formation et emploi seraient plus nettes quand le système éducatif est standardisé sur tout le territoire, et "stratifié ", c'est-à-dire organisé en filières nettement hiérarchisées; de même, ces relations seraient plus marquées dans les pays où il existe des formations spécifiques à tel ou tel groupe de professions. Là où ces différentes caractéristiques sont réunies, les niveaux de sortie et de diplôme seraient nettement plus « lisibles " pour les chefs d'entreprise. A l'inverse, et on peut à cet égard opposer la France et l'Allemagne, quand les diplômes n'apparaissent pas clairement hiérarchisés, ni très spécifiques à un groupe de professions, les relations entre formation et emploi s'avèrent plus lâches; une tendance à l'inflation des diplômes est alors couramment observée, puisque le diplôme est plus un indicateur de classement dans la "file d'attente ", et le long d'une hiérarchie unidimensionnelle, qu'un indicateur univoque de compétences.

Au total, on s'attendrait plutôt à ce que les inégalités sociales liées aux choix d'études soient plus discrètes là où les relations formation-emploi ne sont pas trop strictes (les études constituant donc un enjeu moins crucial) ; mais ceci reste largement à vérifier sur le plan empirique (même si les inégalités face à l'école sont effectivement apparues plus faibles en Grande-Bretagne). Une chose est claire, les comparaisons peuvent seules rendre visible l'effet de paramètres institutionnels, en général uniformes au sein d'un pays donné ; elles sont donc dotées d'un pouvoir heuristique incontestable.

\section{DÉMOCRATISATION, DE QUOI PARLE-T-ON ?}

II semble donc que nous soyons, en France de manière particulièrement marquée par rapport aux pays voisins, en présence d'une démocratisation liée à l'ouverture du système, via l'ouverture de l'entrée en $6^{\theta}$ à partir des années 50 et la généralisation des carrières complètes au collège à partir du début des années $90 . \mathrm{Si}$, au total, les variables d'origine sociale apparaissent moins corrélées avec le plus haut niveau éducatif atteint, cette évolution reflète plus cette ouverture du système que des changements significatifs dans ses modes de fonctionnement (bien que des modifications structurelles récentes, telles que le développement des classes passerelles pour accéder au baccalauréat, fût-il professionnel, puissent aller dans le sens d'une démocratisation plus qualitative).

Si c'est l'accès au "bien" éducation qui est jugé essentiel, qu'importe que la démocratisation se fasse par l'ouverture ou par le fonctionnement. On aura alors tendance à se rallier aux conclusions relativement pessimistes de Prost (1986) quant à l'impact des réformes: "les effets "redistributeurs" des politiques scolaires sont faibles quand ces politiques sont seulement qualitatives et qu'elles se contentent de modifier les structures ou les contenus de l'enseignement. Ils sont plus forts, en revanche, quand il s'agit de politiques quantitatives, provoquant la croissance des effectifs. C'est celle-ci qui fait avancer la démocratisation et non les réformes de l'enseignement ".

Mais pour qui souligne que l'éducation n'est pas seulement un "bien de consommation " mais a aussi une valeur instrumentale et distinctive, il n'est pas anodin d'observer, à côté d'une démocratisation par l'ouverture, une stabilité des inégalités inhérentes au déroulement des carrières, avec comme résultante une translation plus qu'une atténuation des inégalités et donc une absence de démocratisation qualitative. Car cette course en avant est sans doute coûteuse aussi bien pour les individus que pour la société (ce point est développé dans Duru-Bellat et Merle, 1997). La prolongation des scolarités peut ainsi apparaître comme une contrainte, corrélative à une certaine dévaluation des diplômes, puisque si les enfants de milieu populaire accèdent à des titres plus élevés, il leur en faut aujourd'hui "plus, pour avoir la même chose". On peut ainsi montrer (Forsé, 1997) que la démocratisation de l'éducation peut être intégralement contrecarrée par une certaine dévaluation des titres, c'est-àdire ne pas déboucher sur une augmentation de la mobilité sociale pour les enfants de milieu populaire, et ce d'autant plus que se maintiennent par ailleurs des relations entre milieu social d'origine et insertion (à diplôme comparable). Mais c'est alors à ce qu'on pourra appeler une démocratisa- 
tion externe que l'on se réfère, externe à l'institution scolaire, qui se trouve en l'occurrence instrumentalisée. La "crise" de l'école moyenne que l'on observe dans la plupart des pays européens ne révélerait-elle pas cette nécessité de refonder, sur d'autres bases que la seule course au rende- ment, chaque jour plus utopique, une expérience scolaire de plus en plus longue?

Marie Duru-Bellat, Université de Bourgogne, IREDU-CNRS Annick Kieffer LASMAS-IDL-CNRS.
(1) Le lecteur intéressé par cette recherche se reportera au cahier IREDU-LASMAS-IdL qui en rend compte (Duru-Bellat et Kieffer, 1999).

(2) Certes, entre 1972 et 1980 , l'écart entre les taux d'accès en $4^{\theta}$ des enfants de cadres et des enfants d'ouvriers a effectivement diminué, comme le met en exergue G. Langouët, puisque les chiffres sont respectivement passés de 95,8 à 97,1 et de 56,3 à 62,7 ; mais ceci vient du fait que les taux des enfants de cadres ne peuvent plus progresser fortement, vu leur proximité de $100 \%$. Si on s'intéresse à la compétition entre ces deux groupes, il convient de calculer le rapport entre les probabilités qu'ont les enfants de ces deux groupes de passer en quatrième plutôt que non, la prise en compte de rapports entre probabilités supprimant cet " effet de plafond": les inégalités "brutes", qui concernent des taux plus élevés et tendent donc à se réduire au fur et à mesure que les couples de taux pris en compte se rapprochent de 100, peuvent en fait ne pas s'atténuer en termes de chances relatives, ce qu'expriment les "odds ratio", qui sont en l'occurrence de 17,7 en 1972 et de 19,7 en 1980 (cf. aussi note suivante).

(3) Cet indicateur, extrêmement courant dans la littérature européenne (et utilisé en France notamment par Euriat et Thélot, 1995) calcule les chances relatives, pour les membres d'un groupe A, d'atteindre tel niveau éducatif plutôt que tel autre, par rapport aux chances qu'ont les membres d'un groupe $B$ d'atteindre ce premier niveau plutôt que le second. II est égal à 1 dans le cas de l'indépendance statistique, c'est à dire quand le rapport de chances d'être scolarisé plutôt que non scolarisé dans une filière ne varie pas d'un groupe à l'autre. Pour reprendre l'exemple précédent, on rapportera le rapport de chances des enfants de cadres d'accéder à une quatrième plutôt que de ne pas y accéder $(95,8 / 100-95,8)$ aux chiffres correspondants pour les enfants d'ouvriers $(56,3 / 100-56,3)$, obtenant ainsi un odds ratio de 17,7 . Le lecteur trouvera un exemple très détaillé de calcul d'odds ratios in Euriat et Thélot, 1995.

(4) Nous n'aborderons pas ici les difficultés rencontrés par le comparatiste du fait de la diversité des nomenclatures utilisées pour caractériser le niveau éducatif atteint, ou encore le milieu social d'origine (cf. Duru-Bellat, Kieffer et MearelliFournier, 1997). Les comparaisons menées sont effectuées dans une nomenclature unique, celle des classes d'Erikson, Goldthorpe et Portocarero.

(5) Un vaste projet comparatif comme le projet CASMIN se fonde sur l'enquête FQP 70 , donc sur des générations nées entre 1910 et 1947, dont les plus jeunes atteignent l'âge d'entrée en $6^{\mathrm{e}}$ avant même la réforme Berthoin. Ces données sont encore utilisées par les comparatistes européens dans les publications du début des années 90 . Relevons que les travaux les plus récents (Brauns, 1998 ; Müller, 1998) menés par des comparatistes allemands utilisent les données récentes et concluent à une démocratisation en France.

(6) Ceci est confirmé, dans nos données, par plusieurs indicateurs : baisse des $R^{2}$ dans les régressions du haut niveau atteint, baisse des coefficients attachés aux caractéristiques familiales dans les modèles expliquant l'accès à différents niveaux éducatifs, mais aussi stabilité voire une hausse de ces mêmes coefficients dans les modèles expliquant l'accès sous conditions d'être entré en $6^{\mathrm{e}}$. En mettant en œuvre une méthode très différente, Duru-Bellat et Mingat (1992) parviennent à une conclusion analogue.

\section{BIBLIOGRAPHIE}

BRAUNS H. (1998). - Bildung in Frankreich. Eine Studie zum Wandel herkunfts- und geschlechtsspezifischen Bildungsverhaltens. Leske + Budrich.

COMBESSIE J-C. (1984). - L'évolution comparée des inégalités: problèmes statistiques. Revue Française de Sociologie, $n^{\circ} 25$, p. 233-254.

DUBET F., MARTUCCELLI D. (1996). - A l'école. Paris : Seuil.

DURU-BELLAT M., MINGAT A. (1992). - Un regard analytique sur la démocratisation de l'enseignement: valeur heuristique et problèmes méthodologiques des comparaisons dans le temps et dans l'espace. Sociétés Contemporaines, $\mathrm{n}^{\circ}$ 11-12, p. 13-30.
DURU-BELLAT M., JAROUSSE J-P., MINGAT A. (1993). - Les scolarités de la maternelle au lycée. Revue Française de Sociologie, XXXIV, p. 43-60.

DURU-BELLAT M., KIEFFER A., FOURNIER-MEARELLI I. (1997). - Le diplôme, l'âge et le niveau: sens et usage dans les comparaisons de systèmes éducatifs. Sociétés Contemporaines, $n^{\circ} 26, p .45-72$.

DURU-BELLAT M., KIEFFER A. (1999). - La démocratisation de l'enseignement "revisitée ": une mise en perspective historique et internationale. Cahier de I'IREDU-LASMAS-IDL (à paraître).

DURU-BELLAT M., MERLE P. (1997). - La démocratisation impossible? Usages sociaux de l'école et 
inégalités sociales des cursus scolaires. Savoir, $n^{\circ} 3-4$, p. 309-331.

ERIKSON R., JONSSON J. (1996). - Can Education Be Equalized ? Londres: Westview Press.

EURIAT M., THELOT C. (1995). - Le recrutement social de l'élite scolaire depuis 40 ans. Education et Formations, $n^{\circ} 41$, p. 3-21.

FORSE M. (1997). - La diminution de l'inégalité des chances scolaires ne suffit pas à réduire l'inégalités des chances sociales. Revue de L'O.F.C.E, $n^{\circ} 63$, p. 229-239.

JONSSON J. (1987). - Class Origin, Cultural Origin, and Educational Attainment: The Case of Sweden. European Sociological Review, vol. 3, $n^{\circ} 3$, p. 229-241.

JONSSON J., MILLS C. (1993). - Social Class and Educational Attainment in Historical Perspective: A Swedish-English Comparison. British Journal of Sociology, vol. $44, n^{\circ} 2$, p. 213-247 (part I) et $n^{\circ} 3$, p. 403-428 (part II).

JONSSON J., MILLS C., MÜLLER W. (1996). - A Half Century of Increasing Educational Openness? Social Class, Gender and Educational Attainment in Sweden, Germany and Britain. In : Erikson et Jonsson (éds.) (op. cit.).

KÖLHER H. (1992). - Bildungsbeteiligung und Sozialstruktur in der Bundesrepublik. Zur Stabilität und Wandel der Ungleichheit von Bildungschancen. Max-Planck-Institut füt Bildungsforschung. Studien und Berichte 53.

LANGOUËT G. (1994). - La démocratisation de l'enseignement aujourd'hui. Paris : ESF.
MARE R.D. (1981). - Change and Stability in Educational Stratification. American Sociological Review, $n^{\circ} 46$, p. $72-87$.

MEAR P., MERLE P. (1992). - “ 1986-1990 : Démocratisation et/ou hiérarchisation scolaire croissante du publics lycéens?"Sociétés Contemporaines, $n^{\circ} 11-12$, p. 31-51.

MERLLIE D. (1985). - L'analyse de l'interaction entre variables: problème statistique ou sociologique? Revue Française de Sociologie, $n^{\circ} 26$, p. 629-652.

MÜLLER W. (1996). - Class Inequalities in Educational Outcomes : Sweden in Comparative Perspective. In Erikson et Jonsson (eds.) (op.cit)

MÜLLER W. (1998). - Erwartete und unerwartete Folgen der Bildungsexpansion. Kölner Zeitschrift für Soziologie und Sozialpsychologie, Sonderheft 38/1998, 81-112.

MÜLLER W., KARLE W. (1993). - Social Selection in Educational Systems in Europe, European Sociological Review, vol. 9, p. 1-23.

MÜLLER W., SHAVIT Y. (1998). - The institutionnal Embeddedness of the Stratification Process. A comparative Study of Qualifications and Occupations in Thirteen Countries. In : Shavit $Y$., et Müller W. (éds.) From School to Work. Oxford : Clarendon Press.

PROST A. (1986). - L'enseignement s'est-il démocratisé ? Paris : PUF.

SHAVIT Y., BLOSSFELD H-P. (éds.) (1993). - Persistent Inequality. Londres : Westview Press. 